\title{
Estimated Prevalence of Glaucoma in South Korea Using the National Claims Database
}

\author{
Sang Jin Seo, Yun Ha Lee, Sang Yeop Lee, Hyoung Won Bae, Samin Hong, \\ Gong Je Seong, and Chan Yun Kim
} Department of Ophthalmology, Severance Hospital, Institute of Vision Research, Yonsei University College of Medicine,
Seoul 120-752, Republic of Korea

Correspondence should be addressed to Chan Yun Kim; kcyeye@yuhs.ac

Received 6 February 2016; Accepted 20 April 2016

Academic Editor: Jesús Pintor

Copyright (c) 2016 Sang Jin Seo et al. This is an open access article distributed under the Creative Commons Attribution License, which permits unrestricted use, distribution, and reproduction in any medium, provided the original work is properly cited.

\begin{abstract}
Purpose. To estimate the prevalence of glaucoma and costs associated with glaucoma care in South Korea between 2008 and 2013 using the Korean national claims database. Design. Retrospective cross-sectional study from a national claims database. Methods. Patients who were diagnosed with glaucoma between 2008 and 2013 were retrospectively identified in the national claims database using glaucoma diagnostic codes. For each year, the prevalence of glaucoma and direct medical costs associated with glaucoma care were estimated. Result. The prevalence of glaucoma in patients $\geq 40$ years of age increased from $0.79 \%$ in 2008 to $1.05 \%$ in 2013 . The number of patients with glaucoma increased by $54 \%$ between 2008 and 2013 ( $9 \%$ average annual increase). The prevalence of glaucoma increased with age and was higher in males than in females. The cost to care for glaucoma patients increased from $\$ 16.5$ million in 2008 to $\$ 29.2$ million in 2013, which translated into an $81 \%$ increase over the 6 years examined (12.7\% average annual increase). Conclusion. The estimated prevalence and socioeconomic burden of glaucoma have steadily increased each year in South Korea. Nevertheless, many glaucoma patients remain undiagnosed in the present study using national claims database.
\end{abstract}

\section{Introduction}

Glaucoma is a chronic eye disease that results in irreversible optic nerve damage, which causes severe vision loss and blindness. It is the second leading cause of blindness worldwide and one of the leading causes of irreversible blindness [1]. The estimated number of people around the world with glaucoma was 64.3 million in 2013 , and this number is expected to increase to 111.8 million in 2040 . The increasing trend in the number of patients with glaucoma will continue because of increased life expectancy. In recently published systematic reviews, the glaucoma prevalence in subjects $>40$ years of age was estimated to be $3.54 \%$ (95\% confidence interval [CI]: 2.09-5.82) globally and 3.40\% (95\% CI: $2.26-$ 5.02) in Asia [2].

Most studies examining the prevalence of glaucoma have been population-based, but these may be limited in determining glaucoma prevalence in real world because of differences in setting of glaucoma diagnosis between clinical practice and population-based studies. In additional, population-based studies varied widely in terms of the eye examination methods and case definitions used [3].

Korean National Health Insurance (NHI) covers 50 million people in South Korea, which represents approximately $97 \%$ of the Korean population. Therefore, we may be able to use this database to obtain accurate statistics on glaucoma in South Korea. These national claims database provides unique real-world data from a large, complete, and unselected population, providing important information about all types of glaucoma disease [3].

In the current study, we estimated the prevalence of glaucoma between 2008 and 2013 in Korea using the national health claims database. This prevalence was then compared to estimates made with previous population-based studies. The direct economic burden of glaucoma in Korea from 2008 to 2013 was also investigated.

\section{Materials and Methods}

All study conduct adhered to the tenets of the Declaration of Helsinki. The Institutional Review Board of Yonsei 
University Health System determined that this study qualified for exempt status (IRB permit number: 2014-2520-001). We used data that was recorded in the national health claims database between 2008 and 2013. Data were obtained from the Health Insurance Review and Assessment (HIRA) service of Korea (http://www.hira.or.kr/), which provided deidentified data for this study. The HIRA is a governmental agency overseen by the Korean Ministry of Health and Welfare that examines and evaluates medical expenses of all citizens (approximately 50 million people; $96.6 \%$ of the population) covered by Korean NHI, the compulsory social insurance, and Medical Aid (approximately 3.4\% of the population). The HIRA database contains payment records from all medical facilities in South Korea and includes information from 5-6 million inpatient visits each year at approximately 1,100 hospitals and 25,000 private clinics [4]. The database contains data on diagnoses, procedures, prescriptions, demographics, and direct medical costs. The HIRA database manages claims using the sixth edition of the Korean Classification of Disease (KCD-6), which is similar to the International Classification of Disease (ICD-10) system. The diagnostic codes for glaucoma are H40 (glaucoma) and H42 (glaucoma in other diseases classified elsewhere). The H40 code family includes diagnoses of glaucoma suspect (or ocular hypertension [OHT], H400), open angle glaucoma (OAG, H401, including normal tension glaucoma [NTG] and primary open angle glaucoma [POAG]), angle closure glaucoma (ACG, H402), secondary glaucoma (H403-406), other glaucoma (H408), and unspecified glaucoma (H409). The H42 code family includes diagnoses of glaucoma related to endocrine, nutritional, and metabolic diseases (H420). Surgical codes used to identify patients with glaucoma were those for nonpenetrating filtering surgery (S5040), iridotomy (S5041), filtering surgery (S5042), trabeculectomy (S5043), cyclophotocoagulation (S5044), cyclocryosurgery for glaucoma (S4055), trabeculotomy (S4057), sinusotomy (S4058), and glaucoma implant surgery (S5049).

Data from the HIRA database were used to estimate the size of the glaucoma population in South Korea. From all payment requests between 2008 and 2013, we extracted all requests for inpatient and outpatient services for patients $>40$ years old whose main or secondary cause for the visit was documented with an $\mathrm{H} 40$ or H42 KCD diagnosis code. Patients that were given an H400 diagnosis code (glaucoma suspect or OHT) were excluded from glaucoma prevalence estimations.

For the calculation of glaucoma care cost, we applied the annual KRW- (Korean Won-) USD (US dollar) exchange rate of each year and current KRW for each year was converted to constant KRW (2013) using an annual inflation rate of 3\% (average increase in consumer price index over 6-year period in South Korea). In the glaucoma care cost, all payment requests for glaucoma diagnosis code were included such as diagnostic test, procedure, and prescriptions.

The annual prevalence of glaucoma was calculated as the number of people who were diagnosed with glaucoma divided by the number of people that received NHI coverage that year. The prevalence of glaucoma was based on patients $>40$ years of age and was examined by age and sex. The $95 \%$
CI of the prevalence rate was estimated based on a Poisson distribution. Linear trend analyses were performed using Mantel-Haenszel tests. All statistical analyses were performed using SPSS statistical software (ver. 19.0; SPSS, Inc., Chicago, IL, USA). Statistical significance was defined as a $P$ value of $<0.05$.

\section{Results}

Among the general population that was at least 40 years old in South Korea, about 30\% visited an ophthalmologist at least once a year. This totaled approximately 7.0 million people in 2008 and 9.6 million in 2013. The numbers of patients with glaucoma increased by approximately $9 \%$ each year $(54.15 \%$ increase between 2008 and 2013) and in 2013, more than 270,000 Koreans visited the hospital for glaucoma. The total cost of glaucoma care in 2013 was over \$29.8 million (USD) and increased by approximately $81 \%$ between 2008 and 2013 . The cost of glaucoma care per person per year increased from approximately $\$ 93.6$ in 2008 to $\$ 110$ in 2013 (Table 1). The estimated prevalence of glaucoma had a significantly increasing trend between 2008 and 2013 (0.796\% in 2008, $0.835 \%$ in $2009,0.883 \%$ in $2010,0.979 \%$ in $2011,1.006 \%$ in 2012, and $1.056 \%$ in 2013, $P<0.001$, Mantel-Haenszel test for linear trend, Table 2). The estimated prevalence of glaucoma suspects was $0.678 \%$ in $2008,0.738 \%$ in $2009,0.814 \%$ in $2010,1.041 \%$ in $2011,1.136 \%$ in 2012 , and $1.206 \%$ in 2013 . The prevalence of glaucoma among people who visited an eye clinic was $2.53 \%$ in $2008,2.53 \%$ in $2009,2.49 \%$ in $2010,2.65 \%$ in $2011,2.71 \%$ in 2012 , and $2.82 \%$ in 2013 (Table 1).

The most common glaucoma subtype was OAG, which accounted for $56-62 \%$ of all glaucoma cases. The prevalence of OAG, including NTG, was $0.459 \%$ (57.6\% OAG) in 2008, $0.475 \%$ (56.9\% OAG) in 2009, $0.497 \%$ (56.3\% OAG) in 2010, $0.574 \%(58.6 \%$ OAG) in 2011, $0.606 \%(60.2 \%$ OAG) in 2012, and $0.651 \%(61.6 \% \mathrm{OAG})$ in 2013 . The proportion of patients with ACG was $8-12 \%$. The prevalence of ACG was $0.093 \%$ (11.7\% ACG), in 2008, 0.095\% (11.4\% ACG) in 2009, $0.1 \%$ (11.3\% ACG) in 2010, 0.095\% (9.7\% ACG) in 2011, $0.090 \%$ (8.9\% ACG) in 2012, and $0.090 \%$ (8.6\% ACG) in 2013. The OAG/ACG ratio was 4.94 in 2008, 5.00 in 2009, 5.00 in 2010, 6.04 in 2011, 6.75 in 2012, and 7.21 in 2013 (Table 2).

The prevalence of glaucoma increased with age. In 2013, the glaucoma prevalence was $2.35 \%$ in subjects $>80$ years old, $2.68 \%$ in subjects $70-79$ years old, $1.66 \%$ in subjects $60-$ 69 years old, $0.70 \%$ in subjects $50-59$ years old, and $0.35 \%$ in subjects 40-49 years old. The prevalence of glaucoma increased annually in each age group but was significantly higher in older age groups ( $>60$ years) than in younger ones (Figure 1). After adjusting for gender, the odds ratio (OR) of developing glaucoma was 1.616-1.759 with each decade increase in age (Table 3). Glaucoma prevalence was higher in males than in females during the time period examined (Table 2). After adjusting for age, males were more likely to have glaucoma than females (OR: 1.131-1.300, Table 3).

The total number and cost of glaucoma surgeries increased annually, with 9773 glaucoma surgeries performed in 2009, 9541 surgeries performed in 2010, 10,061 surgeries performed in 2011, 10,912 surgeries performed in 2012, and 
TABLE 1: Glaucoma prevalence and glaucoma care costs between 2008 and 2013 in Koreans over 40 years of age.

\begin{tabular}{|c|c|c|c|c|c|c|}
\hline & 2008 & 2009 & 2010 & 2011 & 2012 & 2013 \\
\hline Total population & $22,092,004$ & $22,835,230$ & $23,555,512$ & $24,315,896$ & $24,988,953$ & $25,678,881$ \\
\hline Population visiting eye clinic & $6,958,082$ & $7,547,494$ & $8,360,447$ & $8,984,627$ & $9,284,879$ & $9,615,033$ \\
\hline $\begin{array}{l}\text { Number of glaucoma patients (\% } \\
\text { glaucoma patients) }\end{array}$ & $175,899(2.53)$ & $190,651(2.53)$ & $207,986(2.49)$ & $238,124(2.65)$ & $251,468(2.71)$ & $271,141(2.82)$ \\
\hline Male & 86,534 & 93,940 & 103,066 & 117,578 & 123,814 & 133,914 \\
\hline Female & 89,365 & 96,711 & 104,920 & 120,546 & 127,654 & 137,227 \\
\hline Glaucoma care costs (millions) & $\$ 16.5$ & $\$ 19.3$ & $\$ 21.7$ & $\$ 22.9$ & $\$ 27.2$ & $\$ 29.8$ \\
\hline Annual glaucoma care costs per person & $\$ 93.6$ & $\$ 101.1$ & $\$ 104.3$ & $\$ 96.2$ & $\$ 108.0$ & $\$ 110.0$ \\
\hline
\end{tabular}

Results based upon data from the national claims database. The 2008-2013 Glaucoma care cost in 2013 constant dollars, using 3\% average annual inflation rate and applying the annual KRW/USD exchange rate of each year.

TABLE 2: Estimated prevalence (\%) of glaucoma in Koreans over 40 years of age.

\begin{tabular}{lcccccc}
\hline & 2008 & 2009 & 2010 & 2011 & 2012 & 2013 \\
\hline OAG (95\% CI) & 0.46 & 0.48 & 0.50 & 0.57 & 0.61 & 0.65 \\
& $(0.456-0.462)$ & $(0.473-0.478)$ & $(0.494-0.500)$ & $(0.570-0.577)$ & $(0.603-0.609)$ & $(0.648-0.654)$ \\
ACG (95\% CI) & 0.093 & 0.095 & 0.100 & 0.095 & 0.090 & 0.090 \\
& $(0.092-0.094)$ & $(0.094-0.096)$ & $(0.099-0.101)$ & $(0.094-0.096)$ & $(0.090-0.091)$ & $(0.089-0.091)$ \\
Overall (95\% CI) & 0.80 & 0.83 & 0.88 & 0.98 & 1.01 & 1.06 \\
Overall (male/female) & $0.793-0.800)$ & $(0.831-0.839)$ & $(0.879-0.887)$ & $(0.975-0.983)$ & $(1.002-1.010)$ & $(1.052-1.060)$ \\
Proportion of OAG & $57.6 \%$ & $0.86 / 0.82$ & $0.91 / 0.86$ & $1.00 / 0.96$ & $1.03 / 0.99$ & $1.08 / 1.03$ \\
Proportion of ACG & $11.7 \%$ & $56.9 \%$ & $56.3 \%$ & $58.6 \%$ & $60.2 \%$ & $61.7 \%$ \\
OAG/ACG ratio & 4.94 & $11.4 \%$ & $11.3 \%$ & $9.7 \%$ & $8.9 \%$ & $8.6 \%$ \\
\hline
\end{tabular}

Data obtained from the national claims database. $\mathrm{OAG}=$ open angle glaucoma, $\mathrm{ACG}=$ angle closure glaucoma, and $\mathrm{CI}=$ confidence interval.

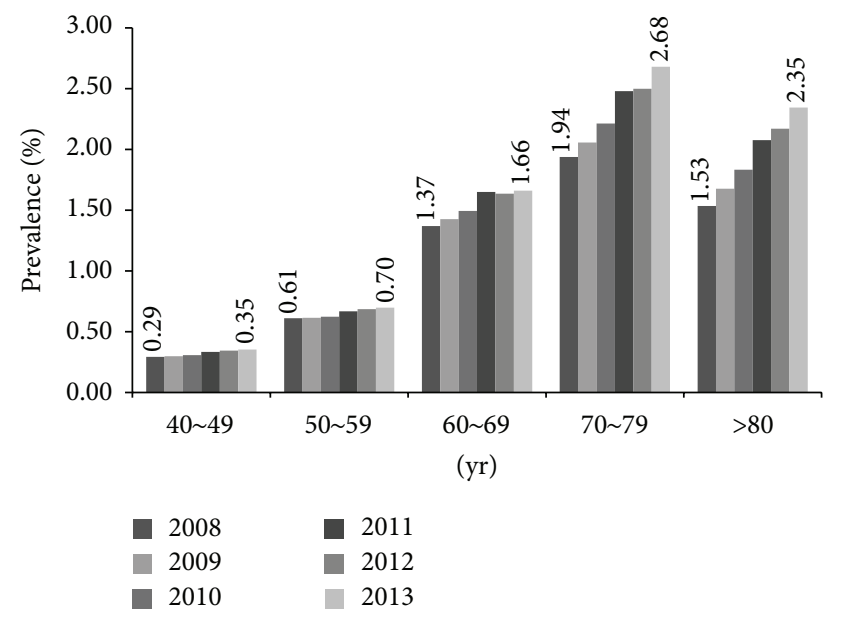

FIGURE 1: Prevalence of glaucoma in each age group. The prevalence of glaucoma significantly increased with age in Korean adults. The trend of increasing prevalence was statistically significant in older (>60 years) groups.

12,502 surgeries performed in 2013. The most common type of glaucoma surgery performed (including laser procedures) was iridotomy, which accounted for $54.9 \%$ of surgeries in $2009,55.4 \%$ of surgeries in $2010,54.4 \%$ of surgeries in 2011 , $53.2 \%$ of surgeries in 2012, and $49.6 \%$ of surgeries in 2013 .
Trabeculectomy was the next most common surgery and accounted for $23.6 \%$ of surgeries in $2009,22.6 \%$ of surgeries in $2010,24.7 \%$ of surgeries in $2011,28.1 \%$ of surgeries in 2012 , and $32.1 \%$ of surgeries in 2013 . The third most common surgery was glaucoma implant surgery, which accounted for $8.5 \%$ of surgeries in $2009,9.9 \%$ of surgeries in $2010,9.2 \%$ of surgeries in 2011, 9.1\% of surgeries in 2012, and $8.1 \%$ of surgeries in 2013. The proportion of iridotomies decreased over time, while that of trabeculectomies increased each year. The cost for all glaucoma surgeries reached approximately $\$ 2.88$ million in 2013 and the proportion of total glaucoma care costs that were related to glaucoma surgeries significantly decreased from $11.36 \%$ in 2009 to $9.14 \%$ in 2013 (Figure 2, Table 4).

\section{Discussion}

We assessed the estimated prevalence of glaucoma and the trend in glaucoma-associated figures in Korea over 6 years (2008 to 2013) using the national claims database. Advantages of using the national claims database over population surveys include its very large sample size and its more representative diagnostic patterns. Korean NHI is compulsory social insurance that covers 50 million Korean people (approximately $97 \%$ of population). It is more powerful than any other national claims database. Additionally, claims data are not regional, but nationwide, which provide more accurate 
TABLE 3: Effect of age and gender on the prevalence of glaucoma.

\begin{tabular}{|c|c|c|c|c|c|}
\hline & & Univar & & Multivar & \\
\hline & & Odds ratio $(95 \% \mathrm{CI})$ & $P$ value & Odds ratio $(95 \% \mathrm{CI})$ & $P$ value \\
\hline & Age, per decade increase & $1.664(1.658-1.670)$ & $<0.001$ & $1.616(1.610-1.622)$ & $<0.001$ \\
\hline 2008 & Sex & & $<0.001$ & & $<0.001$ \\
\hline 2000 & Female & 1.000 (reference) & & 1.000 (reference) & \\
\hline & Male & $1.047(1.037-1.056)$ & & $1.131(1.120-1.142)$ & \\
\hline & Age, per decade increase & $1.683(1.678-1.689)$ & $<0.001$ & $1.759(1.753-1.766)$ & $<0.001$ \\
\hline 2009 & Sex & & $<0.001$ & & $<0.001$ \\
\hline & Female & 1.000 (reference) & & 1.000 (reference) & \\
\hline & Male & $1.048(1.038-1.057)$ & & $1.300(1.288-1.311)$ & \\
\hline & Age, per decade increase & $1.704(1.698-1.709)$ & $<0.001$ & $1.723(1.717-1.729)$ & $<0.001$ \\
\hline 2010 & Sex & & $<0.001$ & & $<0.001$ \\
\hline 2010 & Female & 1 (reference) & & 1.000 (reference) & \\
\hline & Male & $1.058(1.048-1.067)$ & & $1.237(1.226-1.248)$ & \\
\hline & Age, per decade increase & $1.719(1.714-1.725)$ & $<0.001$ & $1.738(1.733-1.743)$ & $<0.001$ \\
\hline 2011 & Sex & & $<0.001$ & & $<0.001$ \\
\hline 2011 & Female & 1.000 (reference) & & 1.000 (reference) & \\
\hline & Male & $1.048(1.039-1.056)$ & & $1.228(1.218-1.238)$ & \\
\hline & Age, per decade increase & $1.712(1.707-1.717)$ & $<0.001$ & $1.729(1.724-1.735)$ & $<0.001$ \\
\hline 2012 & Sex & & $<0.001$ & & $<0.001$ \\
\hline 2012 & Female & 1.000 (reference) & & 1.000 (reference) & \\
\hline & Male & $1.040(1.032-1.049)$ & & $1.214(1.204-1.223)$ & \\
\hline & Age, per decade increase & $1.730(1.725-1.735)$ & $<0.001$ & $1.748(1.743-1.753)$ & $<0.001$ \\
\hline 2013 & Sex & & $<0.001$ & & $<0.001$ \\
\hline 2013 & Female & 1.000 (reference) & & 1.000 (reference) & \\
\hline & Male & $1.046(1.039-1.054)$ & & $1.223(1.214-1.232)$ & \\
\hline
\end{tabular}

$\mathrm{CI}=$ confidence interval.

TABLE 4: The cost and number of glaucoma surgeries (including laser procedures) in Korea between 2009 and 2013 (2008 database was unavailable).

\begin{tabular}{lccccc}
\hline & 2009 & 2010 & 2011 & 2012 & 2013 \\
\hline Cost of glaucoma surgeries (US\$) & $2,226,234$ & $2,148,179$ & $2,211,149$ & $2,414,895$ & $2,847,254$ \\
\hline Iridectomy (\%) & $5,362(54.9 \%)$ & $5,288(55.4 \%)$ & $5,469(54.4 \%)$ & $5,809(53.2 \%)$ & $6,202(49.6 \%)$ \\
Trabeculectomy (\%) & $2,301(23.4 \%)$ & $2,152(22.6 \%)$ & $2,489(24.7 \%)$ & $3,067(28.1 \%)$ & $4,013(32.1 \%)$ \\
Glaucoma implant surgery (\%) & $831(8.5 \%)$ & $946(9.9 \%)$ & $928(9.2 \%)$ & $991(9.1 \%)$ & $1,009(8.1 \%)$ \\
Iris, ciliary photocoagulation & 822 & 668 & 779 & 614 & 205 \\
Trabeculotomy & 236 & 279 & 93 & 95 & 365 \\
Ciliary body cryotherapy & 96 & 86 & 31 & 38 & 89 \\
Nonpenetrating filtering surgery & 63 & 47 & 64 & 58 & 80 \\
Other filtering surgery & 57 & 73 & 10,061 & & 10,912 \\
Total number of surgeries & 9,773 & 9,541 & & 12,502 \\
\hline
\end{tabular}

Results based on data from the national claims database.

estimates of disease prevalence in Korea. Because claims data are longitudinal, they can also be used to recognize trends over time in disease prevalence.

In the present study, the number of patients with glaucoma significantly increased each year (54\% increase between 2008 and 2013). Tham et al. [2] estimated that the number of people worldwide with glaucoma will increase over 2013 levels by $18.3 \%$ in 2020 and by $74 \%$ in 2040 , largely because of an increased life expectancy. In our study, the proportion of elderly patients (>60 years) in the Korean NHI database gradually increased from $14.28 \%$ in 2008 to $16.88 \%$ in 2013 . This was not enough to explain the marked increase we observed in the incidence of glaucoma, which was higher than in previous studies $[1,2]$.

It is possible that the dramatic increase in people with glaucoma observed here does not reflect an increase in glaucoma prevalence. Rather, the glaucoma increase may reflect improvements in glaucoma detection, which may have 
TABLE 5: Estimated prevalence of undetected glaucoma.

\begin{tabular}{|c|c|c|c|c|c|c|}
\hline & 2008 & 2009 & 2010 & 2011 & 2012 & 2013 \\
\hline Population visiting an eye clinic & $6,958,082$ & $7,547,494$ & $8,360,447$ & $8,984,627$ & $9,284,879$ & $9,615,033$ \\
\hline $\begin{array}{l}\text { Number of patients diagnosed with glaucoma } \\
\text { (from national claims database) }\end{array}$ & 175,899 & 190,651 & 207,986 & 238,124 & 251,468 & 271,141 \\
\hline Estimated number of glaucoma patients ${ }^{\mathrm{a}}$ & 773,220 & 799,233 & 824,443 & 851,056 & 874,613 & 898,761 \\
\hline $\begin{array}{l}\text { Estimated number of patients with undetected } \\
\text { glaucoma }{ }^{\mathrm{b}}\end{array}$ & 529,687 & 535,071 & 531,827 & 536,594 & 549,643 & 562,235 \\
\hline Proportion of patients with undetected glaucoma & $68.5 \%$ & $66.9 \%$ & $64.5 \%$ & $63.0 \%$ & $62.8 \%$ & $62.5 \%$ \\
\hline
\end{tabular}

Results based upon data from the national claims database. ${ }^{a}$ Estimated value based on the $3.5 \%$ glaucoma prevalence reported in a systematic review [2] (i.e., total population $\times 0.035$ ). ${ }^{b}$ Estimated value based on the population who had not been to an eye clinic within a year (i.e., $0.35 \times$ [total population - population who visited an eye clinic within one year]).

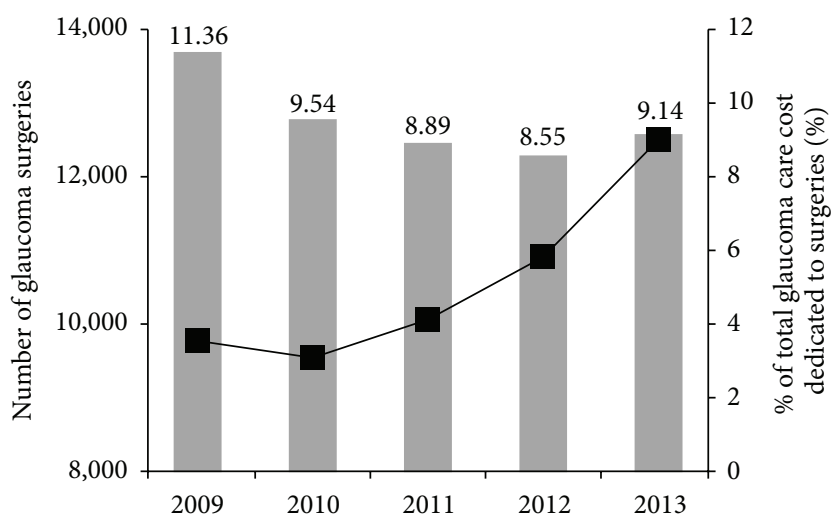

FIGURE 2: Trends in glaucoma surgery costs and number of procedures in Korea. The number of glaucoma surgeries performed (black squares) increased annually, but the proportion of total glaucoma care costs associated with glaucoma surgery (gray bars) decreased annually. Both trends were statistically significant.

occurred for several reasons. First, access to eye healthcare improved during the study period. Between 2008 and 2013 , the number of eye clinics in Korea increased by $9 \%$ (1301 clinics in 2008 and 1418 clinics in 2013) and the number of ophthalmologists increased by $23 \%$ (determined from NHI database). The increase in refractive and cataract surgery also led to improvements in glaucoma detection in Korea. In almost all eye clinics, preoperative ophthalmic examinations include fundus and optic nerve examinations. Therefore, the higher surgical rate increased the chance of identifying patients with asymptomatic glaucoma. Second, the national health education and health promotion program has increased interest and awareness about health and disease in Korea. Additionally, the development of the internet and multimedia has improved disease awareness among the general population. Informing the community about glaucoma is an important step in promoting preventive ophthalmic care [5]. In Korea, news stories related to glaucoma significantly increased from 533 stories in 2008 to 1490 stories in 2013 (data obtained from the internet portal site, NAVER [http://www.naver.com/]). Additionally, the Korean national health check-up program does not include a fundus examination, but most hospitals have implemented programs that include fundus examinations as part of medical checkups. Therefore, more fundus examinations were performed as the population received more recent medical check-ups, which subsequently resulted in more glaucoma diagnoses.

Several studies have reported the prevalence of glaucoma in East Asia and in South Korea. Kim et al. [6] found that the prevalence of glaucoma in rural populations over 50 years of age was 3.4\% (95\% CI: $2.1 \%-4.8 \%$ ). The Namil Study Group performed a population-based survey of glaucoma in a rural area of central South Korea and found that the prevalence of glaucoma was 4.5\% [7]. Similarly, the Tajimi Study Report $[8,9]$ reported a glaucoma prevalence of $5.0 \%$ (95\% CI: $4.2 \%-$ $5.8 \%)$ in central Japan and a systematic review reported a glaucoma prevalence of $2.26 \%-5.02 \%$ in Asia [2]. Although the prevalence of glaucoma among the population that visited an eye clinic was similar to other reports (2.53-2.82\%), we found a much lower estimated prevalence of glaucoma among the total population $(1.06 \%)$, as well as a lower POAG prevalence $(0.65 \%)$, in 2013 based on the national claims database. We speculate that our glaucoma prevalence was underestimated, even though disease incidence can be overestimated when using national claims data $[10,11]$.

Other population-based studies found a substantially higher prevalence of glaucoma than reported in the current study. Our study may have underestimated glaucoma prevalence for several reasons. First, the most common type of glaucoma is chronic asymptomatic disease. Because glaucoma can remain asymptomatic until it becomes severe, there is a high likelihood that the number of affected individuals is much higher than the number known to have the disease $[12,13]$. In fact, several studies have reported a very low awareness of and knowledge about glaucoma in the general population $[5,14]$, with only $10-50 \%$ of people with glaucoma aware that they have the disease [12,13, 15-17]. Additionally, the high rate of undiagnosed POAG (92.7\%) was shown in a population-based study in Korea [18]. When we estimate the number of patients with glaucoma using the $3.5 \%$ glaucoma prevalence reported in a systematic review [2], the proportion of undetected patients with glaucoma in our study was about $70 \%$ (Table 5). Only patients already diagnosed with glaucoma were included in our analyses. Therefore, the chronic characteristics of glaucoma may be well reflected. Moreover, our underestimated prevalence of glaucoma may have resulted from the high proportion of NTG in Korea. 
Although elevated intraocular pressure (IOP) is the hallmark sign and a well-known risk factor for glaucoma, several population-based studies found that IOP was $<22 \mathrm{mmHg}$ in $25-50 \%$ of glaucoma patients [19]. In fact, $77 \%$ of patients with OAG in Korea have NTG [18]. Unfortunately, NTG cannot be detected through tonometry. Therefore, it is more difficult to detect than OAG with ocular hypertension.

Even though our estimated prevalence of diagnosed glaucoma was lower than in other population-based studies, the features of glaucoma prevalence were similar. In agreement with previous studies $[2,3,18,20]$, our multivariate analysis revealed a gender-adjusted OR for glaucoma of 1.7 with each decade increase in age. Additionally, glaucoma was more prevalent in males than in females after adjusting for age (OR: 1.1-1.3). Some controversy remains about whether gender affects glaucoma prevalence, but previous studies conducted in Korea have also shown a higher prevalence in males $[6,20]$. In our multivariate analysis model, age had a more significant effect on glaucoma prevalence than gender (Table 3). Additionally, the fact that OAG and ACG accounted for the highest and second highest proportion of glaucoma cases, respectively, is consistent with other studies [2, 20,21]. We found that the OAG/ACG ratio was 4.9-7.2 (Table 2), which is also similar to other studies conducted on East Asian populations $[7,21]$.

Both the number of glaucoma cases and the number of glaucoma surgeries showed an increasing trend over time. Interestingly, the proportion of money spent on glaucoma surgeries decreased annually (Figure 2). This may have been caused by a progressive increase in antiglaucoma medication usage.

Our study had several imitations. First, people with undiagnosed glaucoma were not included in our estimates of glaucoma prevalence, which may have resulted in an underestimated prevalence. Additionally, national claims data do not always match hospital chart records. It has been shown that national claims data and hospital records only have a 70$80 \%$ concordance rate [22]. Second, although data from the national claims database better represents the clinical situation than data from population-based studies, inconsistencies between clinicians in disease diagnostic criteria and practical patterns may have influenced our results, especially for angle closure glaucoma. Finally, we did not assess risk factors for glaucoma (e.g., myopia, elevated IOP, and comorbid conditions), which would require further investigation with additional national claims data.

In conclusion, the prevalence and socioeconomic burden of glaucoma are increasing annually. Nevertheless, based on national claims data, we speculate that many patients with glaucoma remain undiagnosed in Korea. Ways to identify these patients are needed so that the disease can be managed to minimize vision loss in glaucoma patients with a long life expectancy.

\section{Competing Interests}

The authors declare that they have no competing interests and report no proprietary or commercial interest in any product mentioned or concept discussed in this paper.

\section{Acknowledgments}

The authors gratefully acknowledge Hye Sun Lee (Medical Research Support section, Yonsei University College of Medicine, Seoul, Korea) for assisting with statistical analyses.

\section{References}

[1] H. A. Quigley and A. T. Broman, "The number of people with glaucoma worldwide in 2010 and 2020," British Journal of Ophthalmology, vol. 90, no. 3, pp. 262-267, 2006.

[2] Y.-C. Tham, X. Li, T. Y. Wong, H. A. Quigley, T. Aung, and C.Y. Cheng, "Global prevalence of glaucoma and projections of glaucoma burden through 2040: a systematic review and metaanalysis," Ophthalmology, vol. 121, no. 11, pp. 2081-2090, 2014.

[3] H. Levkovitch-Verbin, I. Goldshtein, G. Chodick, N. Zigman, and V. Shalev, "The maccabi glaucoma study: prevalence and incidence of glaucoma in a large Israeli health maintenance organization," American Journal of Ophthalmology, vol. 158, no. 2, pp. 402-408.e1, 2014.

[4] H.-A. Kim, S. Kim, Y. I. Seo et al., "The epidemiology of total knee replacement in South Korea: national registry data," Rheumatology, vol. 47, no. 1, pp. 88-91, 2008.

[5] K. Mansouri, S. Orgül, F. Meier-Gibbons, and A. Mermoud, "Awareness about glaucoma and related eye health attitudes in Switzerland: a survey of the general public," Ophthalmologica, vol. 220, no. 2, pp. 101-108, 2006.

[6] J. H. Kim, S. Y. Kang, N. R. Kim et al., "Prevalence and characteristics of glaucoma among Korean adults," Korean Journal of Ophthalmology, vol. 25, no. 2, pp. 110-115, 2011.

[7] Y. Y. Kim, J. H. Lee, M. D. Ahn, and C. Y. Kim, "Angle closure in the Namil study in central South Korea," Archives of Ophthalmology, vol. 130, no. 9, pp. 1177-1183, 2012.

[8] A. Iwase, Y. Suzuki, M. Araie et al., "The prevalence of primary open-angle glaucoma in Japanese: the Tajimi Study," Ophthalmology, vol. 111, no. 9, pp. 1641-1648, 2004.

[9] T. Yamamoto, A. Iwase, M. Araie et al., "The Tajimi study report 2: Prevalence of primary angle closure and secondary glaucoma in a Japanese population," Ophthalmology, vol. 112, no. 10, pp. 1661-1669, 2005.

[10] J. D. Stein, T. S. Blachley, and D. C. Musch, "Identification of persons with incident ocular diseases using health care claims databases," American Journal of Ophthalmology, vol. 156, no. 6, pp. 1169-e3, 2013.

[11] J. D. Stein, L. M. Niziol, D. C. Musch et al., "Longitudinal trends in resource use in an incident cohort of open-angle glaucoma patients: resource use in open-angle glaucoma, American Journal of Ophthalmology, vol. 154, no. 3, pp. 452-459.e2, 2012.

[12] M. T. Leite, L. M. Sakata, and F. A. Medeiros, "Managing glaucoma in developing countries," Arquivos Brasileiros de OftaImologia, vol. 74, no. 2, pp. 83-84, 2011.

[13] A. P. Rotchford, J. F. Kirwan, M. A. Muller, G. J. Johnson, and P. Roux, "Temba glaucoma study: a population-based crosssectional survey in urban South Africa," Ophthalmology, vol. 110, no. 2, pp. 376-382, 2003.

[14] M. Katibeh, H. Ziaei, E. Panah et al., "Knowledge and awareness of age related eye diseases: A population-based survey," Journal of Ophthalmic and Vision Research, vol. 9, no. 2, pp. 223-231, 2014.

[15] A. Hennis, S.-Y. Wu, B. Nemesure, R. Honkanen, and M. C. Leske, "Awareness of incident open-angle glaucoma in a population study: the barbados eye studies," Ophthalmology, vol. 114, no. 10, pp. 1816-1821, 2007. 
[16] R. V. Sathyamangalam, P. G. Paul, R. George et al., "Determinants of glaucoma awareness and knowledge in urban Chennai," Indian Journal of Ophthalmology, vol. 57, no. 5, pp. 355-360, 2009.

[17] D. L. Budenz, K. Barton, J. Whiteside-De Vos et al., "Prevalence of glaucoma in an urban west african population: the tema eye survey," JAMA Ophthalmology, vol. 131, no. 5, pp. 651-658, 2013.

[18] C. S. Kim, G. J. Seong, N. H. Lee, and K. C. Song, "Prevalence of primary open-angle glaucoma in central South Korea the Namil study," Ophthalmology, vol. 118, no. 6, pp. 1024-1030, 2011.

[19] R. N. Weinreb, T. Aung, and F. A. Medeiros, "The pathophysiology and treatment of glaucoma: a review," The Journal of the American Medical Association, vol. 311, no. 18, pp. 1901-1911, 2014.

[20] K.-C. Yoon, G.-H. Mun, S.-D. Kim et al., "Prevalence of eye diseases in South Korea: data from the Korea national health and nutrition examination survey 2008-2009," Korean Journal of Ophthalmology, vol. 25, no. 6, pp. 421-433, 2011.

[21] H.-K. Cho and C. Kee, "Population-based glaucoma prevalence studies in Asians," Survey of Ophthalmology, vol. 59, no. 4, pp. 434-447, 2014.

[22] I. B. Park, D. J. Kim, J. Kim et al., "Current status of aspirin user in Korean diabetic patients using Korean health insurance database," The Journal of Korean Diabetes Association, vol. 30, no. 5, article 363, 2006. 


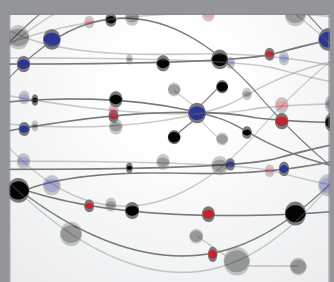

The Scientific World Journal
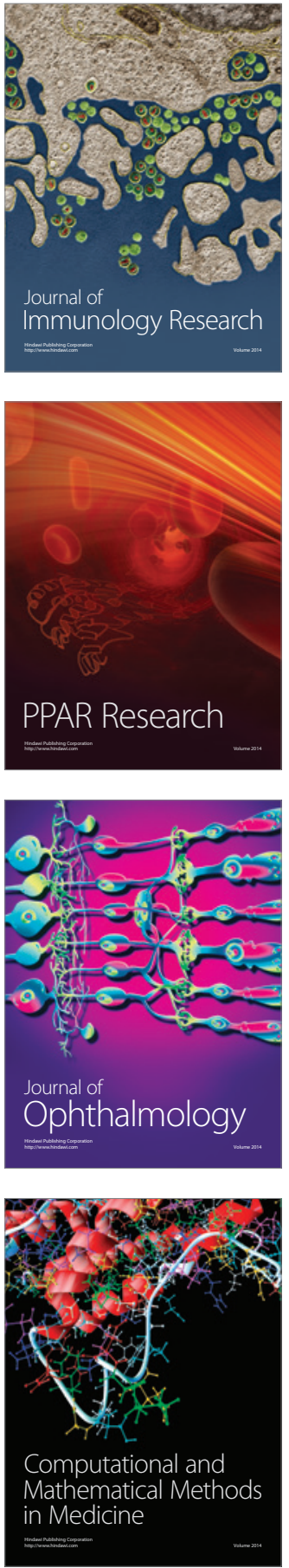

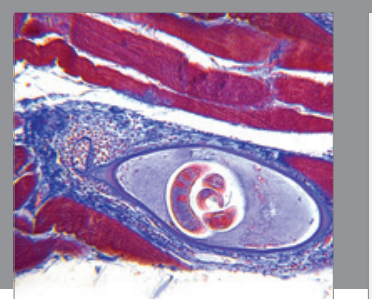

Gastroenterology Research and Practice

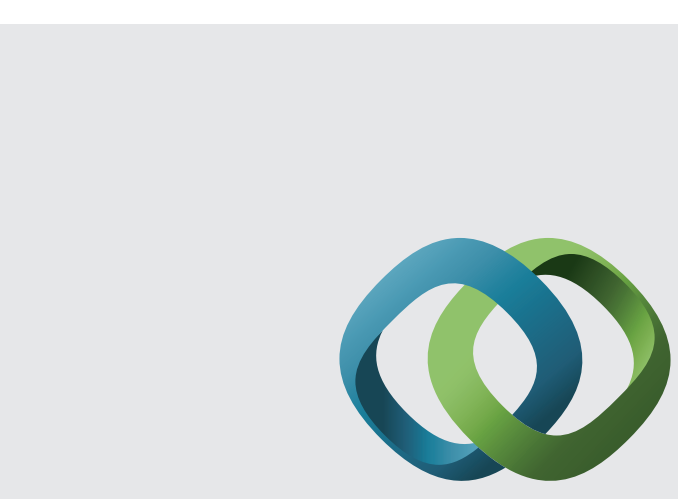

\section{Hindawi}

Submit your manuscripts at

http://www.hindawi.com
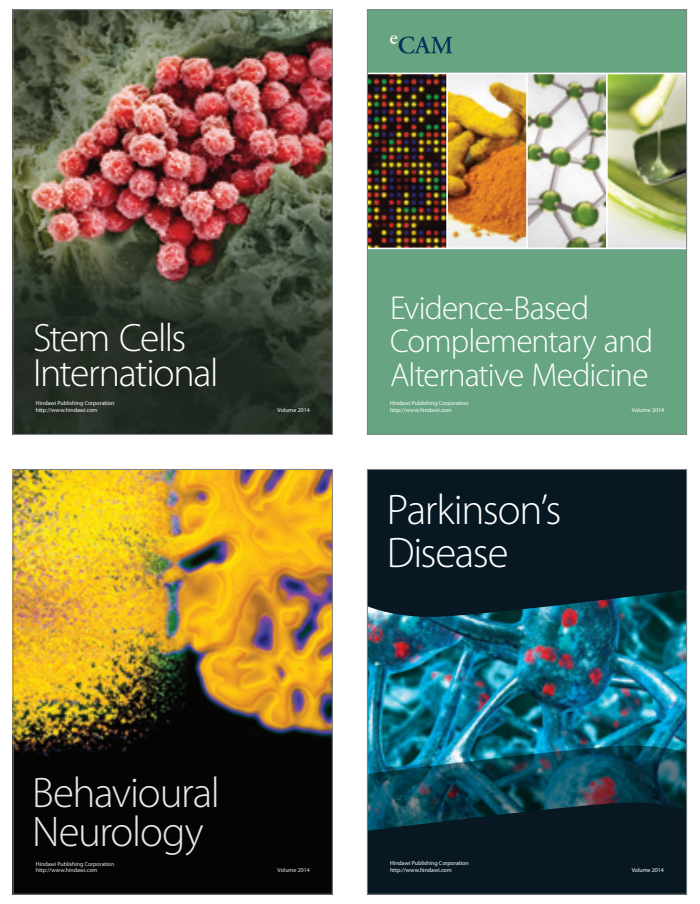
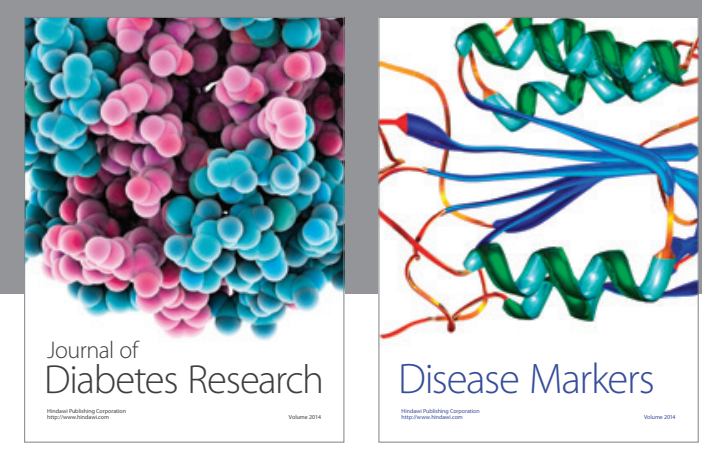

Disease Markers
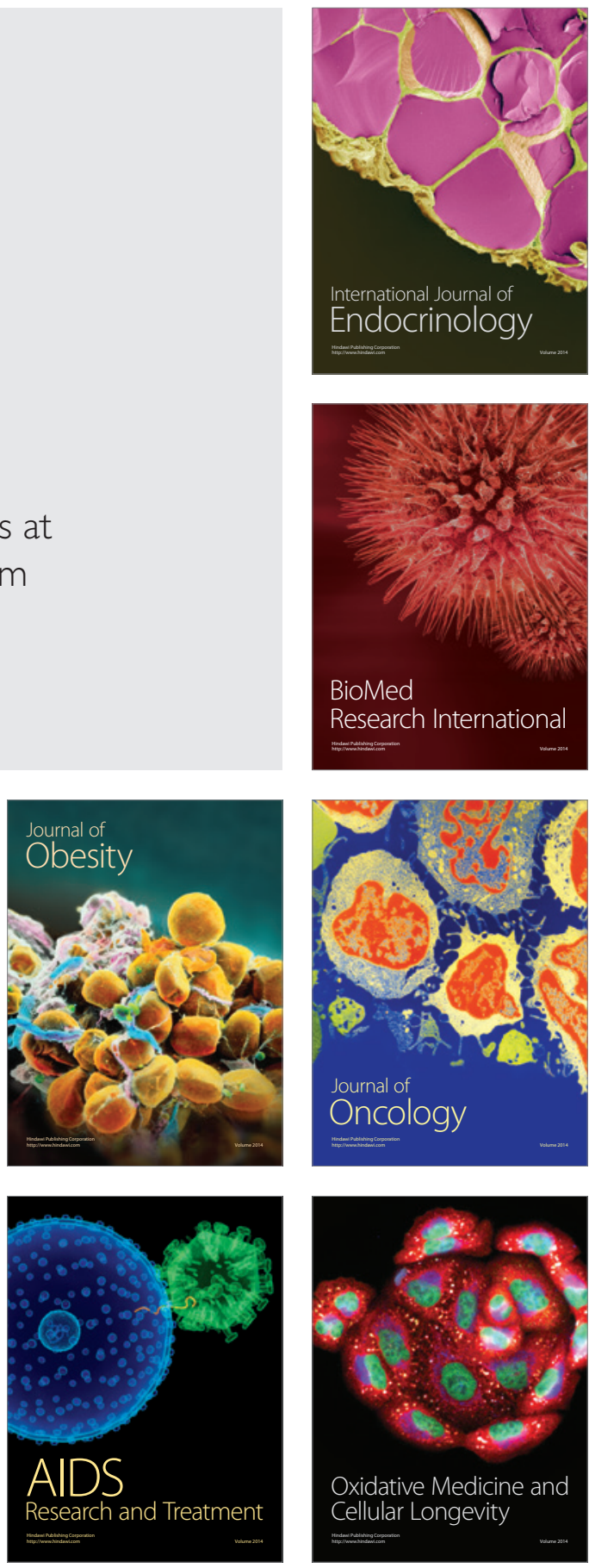